\title{
Soluble tumour necrosis factor receptor I is a promising early indicator of complicated clinical outcome in patients following severe trauma
}

\author{
ANETA M. BINKOWSKA ${ }^{l}$, GRZEGORZ MICHALAK ${ }^{2,3}$, MARIA KOPACZ $^{4}$, ROBERT SŁOTWIŃSKI ${ }^{4,5}$ \\ ${ }^{1}$ Department of Disaster Medicine, Medical University of Warsaw, Warsaw, Poland \\ ${ }^{2}$ Department of Emergency Medicine, Medical University of Warsaw, Warsaw, Poland \\ ${ }^{3}$ Bielański Hospital. Fr. J. Popieluszko in Warsaw, Poland \\ ${ }^{4}$ Department of Immunology, Biochemistry and Nutrition, Medical University of Warsaw, Warsaw, Poland \\ ${ }^{5}$ Mossakowski Medical Research Centre Polish Academy of Sciences, Warsaw, Poland
}

\begin{abstract}
Post-traumatic mortality rates are still very high and show an increasing tendency. Early identification of patients at high risk of severe complications has a significant impact on treatment outcomes. The aim of the study was to better understand the early pathological inflammatory response to injury and infection, and to determine the usefulness of the assessment of TNF- $\alpha$ and sTNFR1 concentrations in the peripheral blood as early indicators of severe post-traumatic complications. The study was carried out in a group of 51 patients after trauma, treated in the ED, including 32 patients who met the inclusion criteria for immunological analysis. Patients were divided into two groups using the ISS scale (A ISS $\geq 20, B$ ISS < 20). The highest TNF- $\alpha$ and sTNFR1 concentrations in both groups were recorded at admission and were significantly higher in group A compared to group $B(A v s . B T N F-\alpha 2.46 \mathrm{pg} / \mathrm{ml}$ vs. $1.78 \mathrm{pg} / \mathrm{ml}$; sTNFR1 $1667.5 \mathrm{pg} / \mathrm{ml} v \mathrm{~s} .875 .2 \mathrm{p}<0.005)$. The concentration of sTNFR1 in patients with severe complications was significantly higher compared to patients without complications and preceded clinical symptoms of complications $\left(C^{+} v s . C^{-} 1561.5 \mathrm{pg} / \mathrm{ml} v \mathrm{~s} .930 .6 \mathrm{pg} / \mathrm{ml}, \mathrm{p}<0,005\right)$. The high diagnostic sensitivity calculated from the ROC curves was found for the concentrations of both cytokines: TNF- $\alpha(A U C=0.91, p=0.004)$ and sTNFR1 $(A U C=0.86, p=0.011)$. Elevated levels of sTNFR1, determined in the peripheral blood shortly after injury, are significantly associated with the occurrence of later complications, which in some patients lead to death. In contrast, high levels of $T N F-\alpha$ shortly after injury are associated with mortality.
\end{abstract}

Key words: sepsis, MODS, inflammation, TNF- $\alpha$, sTNFR1, trauma.

(Centr Eur J Immunol 2019; 44 (4): 423-432)

\section{Introduction}

According to the World Health Organization, post-traumatic mortality rates are still very high and show an increasing tendency. Severe infections and multiple organ dysfunction syndrome (MODS) are among the most severe traumatic complications, burdened with high mortality [1]. Mortality in the course of sepsis complicated by septic shock or multi-organ failure is $25-75 \%[2,3]$. Early systemic inflammatory response syndrome (SIRS) is one of the more important factors leading to MODS, which accompanies severe trauma and infection. Late detection of this reaction, i.e. at the time of clinical symptoms of sepsis or MODS, causes significantly lower effectiveness of ther- apy. As shown by previous studies, increased production of selected mediators of the early inflammatory response (e.g. cytokines) may precede clinical symptoms of severe sepsis and MODS [3, 4]. This is probably related to two phases of the inflammatory response to injury and infection. The simplified scheme of the immune response to injury and infection assumes that the early stage is primarily a pro-inflammatory stage mediated by proinflammatory cytokines (including TNF- $\alpha$, IL-1, and IL-6). The second stage is the anti-inflammatory phase, with increased activity of anti-inflammatory cytokines (IL-1Ra, sTNFR1, IL-4, and IL-10) showing immunosuppressive action and activation of the mechanisms of acquired cellular immunity [5-8].

Correspondence: Aneta M. Binkowska, MA, Department of Disaster Medicine, Medical University of Warsaw, 61A Żwirki i Wigury St., 02-091 Warsaw, Poland, e-mail: aneta.binkowska@wum.edu.pl Submitted: 29.01.2018; Accepted: 30.01.2018 
Most recent genetic research has questioned the presence of two phases in the inflammatory response to trauma and infection, suggesting that the pro- and anti-inflammatory responses proceed in parallel, which further complicates the early diagnostics of severe complications (e.g. MODS) and hinders distinguishing physiological inflammatory response from pathological response [9-11]. Post-traumatic "genetic storm" based on activation of approximately 5000 genes over several tens of hours does not always correlate with increased production of pro- or anti-inflammatory mediators $[10,12]$. Some researchers question the significance of SIRS reactions, while stressing the important contribution of cellular immunity disorders (e.g. increased lymphocyte apoptosis), accompanied by immunosuppression, which is supposed to be the main cause of increased mortality in post-traumatic sepsis [13]. The heterogeneity of the studied patient groups, co-morbidities, and variable biological properties of the mediators, conditioning different effects, may be other factors that hamper the ability to distinguish a pathological inflammatory response from a physiological response, and thus the early diagnostics of severe post-traumatic complications [14].

The size and type of trauma, concomitant infection, and therapeutic treatment (e.g. necessity for emergency surgery, transfusion) are of particular importance in the assessment of the early inflammatory response to injury and infection in patients treated in the hospital emergency department (ED). Scales assessing the severity of the patient's condition, the extent of the injury, and routine laboratory tests have limited sensitivity and specificity in the early assessment of severe post-traumatic complications. The selection of appropriate immunological indicators for early risk assessment of severe complications increasing mortality remains an open problem. It is known that concentrations of cellular response mediators in the peripheral blood (e.g. cytokines) depend, among others, on their half-life, time from the injury, and the presence of their soluble receptors or inhibitors. It is also difficult to assess the quantity and affinity of tissue receptors (e.g. located on endothelial cells) that bind cytokines secreted to the peripheral blood. It involves transduction of different signals to the cell and the lack of correlation between the expression of these receptors and the response to a given cytokine. In addition, concentrations of peripheral blood cytokines may be different than at the site of the injury $[10,15,16]$.

TNF- $\alpha$ is the key cytokine of the inflammatory response to trauma and infection produced predominantly by monocytes and macrophages in response to lipopolysaccharide (LPS) of bacterial walls. The effects of biological action of TNF- $\alpha$ are mainly dependent on the quantity of secreted cytokine. Rapid secretion after major trauma with infection can lead in a short time to shock, acute respiratory failure, intravascular coagulation, high fever, and death. On the other hand, the reduced ability of animals to synthesise TNF- $\alpha$ increases the sensitivity to a lethal dose of Escherichia coli [17]. TNF- $\alpha$ was found to be released faster than oth- er pro-inflammatory cytokines and had a significant effect on the occurrence of immunosuppression. TNF- $\alpha$ exerts its influence on the immune system not only directly, but also by inducing the release of many cytokines [18]. Stimulation of TNF- $\alpha$ receptors may also cause direct tissue damage, macro- and microcirculation disorders with subsequent ischaemia, and/or reperfusion [19]. The effect of TNF- $\alpha$ is dependent on TNF receptors on the cell surface. We currently know two different receptors: sTNFR1 (p55, CD120 $\alpha$ ) and sTNFR2 (p75, CD120 $\beta$ ). These receptors are found in every nucleated cell, mainly on leukocytes and endothelial cells, which may explain the multidirectional action of TNF- $\alpha$ : antibacterial, anticancer, but also in autoimmune diseases or circulatory insufficiency. At high concentrations, sTNFR1 inhibits TNF- $\alpha$ by preventing binding of this cytokine to membrane receptors. The sTNFR1 receptor binds both membrane and soluble forms of TNF- $\alpha$, while sTNFR2 binds mainly the membrane TNF- $\alpha$ [20, 21].

There are a number of hypotheses explaining the mechanisms of organ dysfunction that occur after trauma, regardless of the cause of the injury. One of these hypotheses points to the increased pro- and anti-inflammatory response in the first hours after major trauma, but does not precisely explain the difference between physiological and pathological immune response to trauma [14]. This problem is essential for the treatment from a clinical point of view because early detection of pathological response to trauma and infection may be important in predicting the course of the disease, starting proper diagnostics, and early treatment. In the classical model of the inflammatory response to injury, compensatory anti-inflammatory response syndrome (CARS) leads to a reduction in the severity of SIRS proinflammatory response in order to restore balance between pro- and anti-inflammatory response, but it leads to increased CARS in some patients and exacerbated immunosuppression [9]. Determination the relationship between excessive inflammatory response and increasing immunosuppression remains an unresolved problem [10]. It is still unknown whether early, excessive inflammatory response is a more important factor leading to severe organ complications or the parallel intensification of immunosuppression. The solution to this problem may be important for the efficacy of appropriate therapy (e.g. early immunomodulatory biological therapy), the aim of which, on the one hand, should be to limit the excessive inflammatory response, and on the other, to stimulate the cells regulating the immune response to trauma and to reduce immunosuppression. Efforts to stimulate cellular immunity in critically ill patients with infections are promising [22], while attempts to reduce the inflammatory response to trauma are still not very effective [23]. In order to better assess the risk of severe complications in patients with trauma, there is a need for better understanding of the early immune response mechanisms to trauma, which may determine the further course of the disease. 
This study was aimed at gaining a better understand the mechanisms of early immune response to trauma. Furthermore, based on the parameters studied (TNF- $\alpha$ and sTNFR1), an attempt was made to determine their practical usefulness in the early diagnostics of severe post-traumatic complications.

\section{Material and methods}

The study group comprised 51 patients, including 39 men and 12 women, aged 18-80 years, average age $41.40 \pm 16.53$ years, admitted to the Emergency Department (ED) of the Bielański and Praski hospitals due to trauma. The largest group consisted of patients after traffic accidents with multi-organ injuries. The injury severity score (ISS), which is most commonly used in the international classification of trauma severity, was used to assess the severity of the patients' status [24]. The entire group of patients, depending on the severity of the condition and the extent of injury, was divided into two subgroups: group A ( $n=23$ ) included patients with ISS $\geq 20$, and group B ( $n=28)$ comprised patients with ISS $<20$. This division was adopted due to the specificity of trauma of the studied groups to better assess the impact of the extent of the injury on the investigated immune parameters. It is known that the ISS rate is the total of squares of the three highest scores in the Abbreviated Injury Scale (AIS) for the values of three body areas. According to this scale, major body injury in most publications is defined as $>15$ points on the ISS. It should be emphasised that the mortality of patients with ISS $<15$ was less than $5 \%$, while ISS in the range of 16-24 points increased the mortality rate to $11-14 \%$, and at ISS $>25$ the mortality rate was over $36 \%[25,26]$ (Table 1).

Among the examined group of patients, inclusion criteria for immunological tests were met by 32 patients, including group A $n=20$ (ISS $\geq 20$ ) and group B $n=12$ (ISS < 20) (Table 2).

The study did not qualify patients over 80 years of age, patients after chemo-radiotherapy, patients with pre-existing chronic liver disease (cirrhosis), kidney diseases, diabetes mellitus, chronic inflammatory diseases, and patients chronically treated before admission to the hospital with steroids or nonsteroidal anti-inflammatory drugs. Clinical monitoring involved the following parameters: type of injury and treatment, including the number of operations/reoperations, type and number of complications, length of hospital stay, and duration of respiratory treatment and mortality. Routine laboratory tests were performed in all patients.

\section{Cytokine measurement}

The concentration of TNF- $\alpha$ and STNFR-1 in the peripheral blood serum was examined at the time of admission to the ED (at the time of first blood collection point 0 ) and after 3, 6, 12, and 24 hours of hospitalisation.
The analysis of cytokine concentrations in the peripheral blood plasma was performed based on commercially available ELISA enzymatic immunoassay kits, in accordance with the instructions provided by the manufacturer (Quantikine Immunoassay human TNF- $\alpha$ and human sTNFR1 R\&D Systems Europe Minneapolis, USA). The sample of venous blood was centrifuged at 3200/min for 15 minutes, then plasma was stored at $-80^{\circ} \mathrm{C}$ for further tests. The lower limit of assay sensitivity was $4.4 \mathrm{pg} / \mathrm{ml}$ and $3.0 \mathrm{pg} / \mathrm{ml}$ for TNF- $\alpha$ and sTNFR-1, respectively. Concentration readings were carried out using a DIALAB ELX 808 spectrophotometer and Gen 51.10 software. The concentration of cytokines was evaluated by comparing the absorbance values to a standard curve prepared by measuring the absorbance of samples of known concentration at a wavelength of $\lambda=450 \mathrm{~nm}$. The results are presented as median $\mathrm{pg} / \mathrm{ml}$. The control group consisted of 20 healthy volunteers in a similar age and sex range, in whom cytokine concentrations in the peripheral blood serum was tested once.

\section{Ethics}

The study acquired the consent of the Bioethics Committee of the Medical University of Warsaw. Each patient gave written consent to perform research and access their disease history. All procedures were performed in accordance with the Declaration of Helsinki.

\section{Statistical analysis}

The relationships between the extent of injury and the occurrence of complications as well as the comparison of concentration values of the tested parameters (median, $\mathrm{pg} / \mathrm{ml}$ ) with the standard and between groups were made using the Mann-Whitney $U$ test. The significance of changes in parameter concentrations was tested using the Wilcoxon test. The relationship between the change in concentration over time and the occurrence of complications was evaluated using the chi-square test. The correlation between cytokine concentrations and the extent of injury and selected laboratory tests was verified by the $\rho$-Spearman test. A series of receiver operating characteristic (ROC) curves was carried out in order to assess whether the likelihood of serious complications, including death, can be assessed based on cytokine levels and the ISS. The influence of age and gender on cytokine concentrations was analysed using the independence chi-square test and the Mann-Whitney $U$ test. The level of significance was assumed at $p<0.05$. The analyses were performed using SPSS 13.0 for Windows.

\section{Results}

\section{Clinical part}

In the presented material, the main cause of multiple organ injury was traffic accidents and falls from heights, 
Table 1. Characteristics of the patient groups

\begin{tabular}{|c|c|c|c|}
\hline \multirow[t]{2}{*}{ Parameter } & & \multicolumn{2}{|c|}{ Studied group of patients } \\
\hline & & $\begin{array}{c}\mathbf{A} \\
(n=23)\end{array}$ & $\begin{array}{c}\text { B } \\
(n=28)\end{array}$ \\
\hline \multicolumn{2}{|l|}{ Age, years } & $45.54 \pm 18.8$ & $39.75 \pm 16.36$ \\
\hline \multicolumn{2}{|l|}{$\operatorname{Sex}(M: F)$} & $19: 4$ & $20: 8$ \\
\hline \multirow[t]{5}{*}{ Cause of injury } & Traffic accident & 14 & 7 \\
\hline & Fall from a height & 5 & 5 \\
\hline & Fall & - & 7 \\
\hline & Beating & 2 & 3 \\
\hline & Others & 2 & 6 \\
\hline \multirow[t]{7}{*}{ Type of injury } & Polytrauma & 15 & 1 \\
\hline & Head damage & 4 & 11 \\
\hline & Chest trauma & 1 & 1 \\
\hline & Abdominal injury & - & 3 \\
\hline & The trauma of the upper limbs & - & 6 \\
\hline & Trauma to the lower extremities & 2 & - \\
\hline & Burns & 1 & 6 \\
\hline \multirow{9}{*}{$\begin{array}{l}\text { Treatment } \\
\text { (ad hoc procedure) }\end{array}$} & Surgery: & & \\
\hline & Laparotomy & 3 & - \\
\hline & Splenectomy & 2 & - \\
\hline & Sewing kidney & 1 & - \\
\hline & Orthopaedic surgery (repositions, fixation, stabilisation, prosthetics) & 15 & 12 \\
\hline & Drainage pneumothorax & 3 & - \\
\hline & Respirator & 6 & \\
\hline & Transfusions & 9 & 1 \\
\hline & Other & 17 & 11 \\
\hline \multirow[t]{7}{*}{ Complications } & MODS & 5 & - \\
\hline & Respiratory failure & 5 & - \\
\hline & Infection (general, local) & 4 & 4 \\
\hline & Internal bleeding & 6 & - \\
\hline & Pulmonary (embolism/stroke) & 2 & - \\
\hline & Poorly healing wounds & 3 & 1 \\
\hline & Other (fever, post-traumatic epilepsy) & 10 & 1 \\
\hline \multirow[t]{4}{*}{ Hospitalisation time } & $<24 \mathrm{~h}$ & 5 & 13 \\
\hline & $<1$ day, $>7$ days & 5 & 8 \\
\hline & $<8$ days, $>14$ days & 6 & 5 \\
\hline & $>15$ days & 7 & 2 \\
\hline Death & & 5 & - \\
\hline
\end{tabular}

found in $53 \%$ of patients. Of 51 patients, $33(64.70 \%)$ required hospitalisation for more than 24 hours, including six patients treated in the intensive care unit (ICU). Twelve patients underwent emergency surgeries (splenectomy, exploratory laparotomy, repositions and bone fixation, chest drain), including two patients who required reoperation due to internal bleeding; in 16 patients, surgical procedures were carried out in the deferred mode (bone fixations and repositions). Of all patients, eight required further deferred surgical interventions (repositions or bone fixation). 
Table 2. Clinical characteristics of patients qualified for immunological tests

\begin{tabular}{|c|c|c|c|}
\hline \multirow[t]{2}{*}{ Parameter } & & \multicolumn{2}{|c|}{ Studied group of patients } \\
\hline & & $\begin{array}{c}\text { A } \\
n=20\end{array}$ & $\begin{array}{c}\text { B } \\
n=12\end{array}$ \\
\hline Age, years & & $43.31 \pm 18.71$ & $41.83 \pm 17.89$ \\
\hline $\operatorname{Sex}(M: F)$ & & $16: 4$ & $7: 5$ \\
\hline \multirow[t]{8}{*}{ Type of injury } & Polytrauma & 15 & 1 \\
\hline & Head damage & 2 & 3 \\
\hline & Chest trauma & - & 1 \\
\hline & Abdominal injury & - & - \\
\hline & The trauma of the upper limbs & - & - \\
\hline & Trauma to the lower extremities & 1 & 5 \\
\hline & Burns & 2 & - \\
\hline & Trauma manifold & 1 & 1 \\
\hline \multirow{9}{*}{$\begin{array}{l}\text { Treatment } \\
\text { (ad hoc procedure) }\end{array}$} & Surgery: & & \\
\hline & Laparotomy & 2 & - \\
\hline & Splenectomy & 1 & - \\
\hline & sewing kidney & 1 & - \\
\hline & Orthopaedic surgery (repositions, fixation, stabilisation, prosthetics) & 8 & 7 \\
\hline & Drainage pneumothorax & 3 & - \\
\hline & Respirator & 6 & \\
\hline & Transfusions & 7 & 1 \\
\hline & Other & 17 & 5 \\
\hline \multirow[t]{7}{*}{ Complications } & MODS & 3 & - \\
\hline & Respiratory failure & 5 & - \\
\hline & Infection (general, local) & 4 & 4 \\
\hline & Internal bleeding & 4 & - \\
\hline & Pulmonary (embolism/stroke) & 2 & - \\
\hline & Poorly healing wounds & 3 & 1 \\
\hline & Other (fever, post-traumatic epilepsy) & 9 & 1 \\
\hline \multirow[t]{4}{*}{ Hospitalisation time } & $<24 \mathrm{~h}$ & 5 & 5 \\
\hline & $<1$ day, $>7$ days & 3 & 4 \\
\hline & $<8$ days, $>14$ days & 5 & 2 \\
\hline & $>15$ days & 7 & 1 \\
\hline Death & & 5 & - \\
\hline
\end{tabular}

Patients with complications $\left(\mathrm{C}^{+}\right)$had a significantly higher ISS score than those without complications $\left(\mathrm{C}^{-}\right)$(Table 3 ).

Of the 32 patients who were included in immunological tests, 16 had complications. The highest number of complications was observed in group A (13 patients; $65 \%)$, while in group B (ISS $<20$ points) they occurred in three patients $(25 \%)$ (Table 2). In the group of the most critically ill patients (group A), two patients died within 12 hours of admission to the ED, and the remaining three on the fifth day of treatment. Patients with lighter injuries, after rele- vant medical procedures, were discharged home or transported to other health care units within 12 hours.

There were no statistically significant differences regarding age and gender in comparable patient groups with immunological tests (A vs. B and $\mathrm{C}^{+}$vs. $\mathrm{C}^{-}$). The level of ISS significantly correlated with selected results of laboratory tests only in the group of the most critically ill patients (group A): haematocrit (HCT), haemoglobin (HGB), erythrocyte count (RBC), blood glucose level, and blood $\mathrm{pH}$ value, but only in the first hours of hospitalisation $(p<0.05)$ (Table 4$)$. 
Table 3. The occurrence of complications and ISS score in the whole group of patients

\begin{tabular}{lccccc}
\hline Variable & Complications & Mean & Standard deviation & Test result $\boldsymbol{Z}$ & Severity level $(\boldsymbol{p})$ \\
\hline \multirow{2}{*}{ ISS } & Yes & 27.68 & 13.98 & 3.66 & $<0.001$ \\
\cline { 2 - 4 } & No & 13.96 & 9.81 & & \\
\hline
\end{tabular}

Table 4. Correlation coefficients between the ISS scale of the subjects and the results of the first biochemical blood test in the group with immunological tests

\begin{tabular}{lcc}
\hline \multirow{2}{*}{ Parameter } & \multicolumn{2}{c}{ ISS } \\
\cline { 2 - 3 } & $\rho$-Spearman $(\boldsymbol{r})$ & Severity level $(\boldsymbol{p})$ \\
\hline HCT & -0.39 & 0.022 \\
\hline RBC & -0.38 & 0.025 \\
\hline HGB & -0.44 & 0.008 \\
\hline pH & -0.97 & $<0.001$ \\
\hline Glucose & 0.49 & 0.027 \\
\hline
\end{tabular}

No significant changes were observed in the remaining laboratory tests during the observation. Changes of selected parameters were at similar levels, regardless of the group examined.

\section{Changes in cytokine concentrations}

Assessment of TNF- $\alpha$ concentrations in comparable groups of patients (A vs. B) showed that the baseline values of this cytokine in both groups were not significantly elevated compared to the control group and amounted to $2.46 \mathrm{pg} / \mathrm{ml}$ in group $\mathrm{A}$, and $1.78 \mathrm{pg} / \mathrm{ml}$ in group B $(p>0.05)$. Further observation of the dynamics of response to trauma and complications showed that the highest TNF- $\alpha$ concentrations occurred in the third hour of hospitalisation for group A and amounted to $2.79 \mathrm{pg} / \mathrm{ml}$, while for group B they occurred slightly later, in the sixth hour of hospitalisation, and equalled $2.73 \mathrm{pg} / \mathrm{ml}$. The increase in TNF $\alpha$ concentration was statistically insignifi-

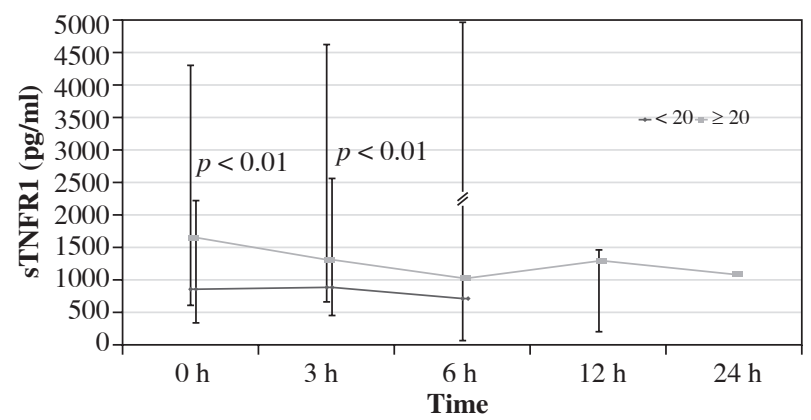

Fig. 1. Concentration of sTNFR1 depending on the extent of the injury (according to ISS score) cant in both groups compared to baseline values. There were no statistically significant differences between the study groups at the time points tested.

Subsequently, the analysis included changes in TNF- $\alpha$ concentrations depending on the existing complications. The concentration of this cytokine in patients with complications did not differ significantly compared to the group without complications (in the third hour of monitoring $\mathrm{C}^{+}$ vs. $\mathrm{C}^{-}: 3.20 \mathrm{pg} / \mathrm{ml}$ vs. $\left.1.93 \mathrm{pg} / \mathrm{ml}, p>0.05\right)$, with significantly higher concentrations in the group of deceased patients only in the baseline examination, at $4.052 \mathrm{pg} / \mathrm{ml}$ $(p<0.05)$. The course of TNF- $\alpha$ curves in both groups differed. The highest increase in the group with complications was recorded after three hours of hospitalisation, while in the group without complications after six hours of monitoring. The concentrations of TNF- $\alpha$ from the sixth hour of observation were at similar levels in both groups (6 hours for $\mathrm{C}^{+} 2.08 \mathrm{pg} / \mathrm{ml}$ and $2.52 \mathrm{pg} / \mathrm{ml}, p=0.364$ for $\mathrm{C}^{-}$).

Evaluation of sTNFR1 concentrations in comparable groups of patients (A vs. B) showed that baseline sTNFR1 concentrations in group A (ISS > 20) were significantly elevated at the time of admission to the ED compared to the standard, at 1667.5 vs. $1060.85 \mathrm{pg} / \mathrm{ml}(p<0.001)$, and they persisted at a significantly elevated level until the sixth hour of observation. Baseline concentrations of this cytokine in group B were significantly lower compared to standard, at $875.2 \mathrm{pg} / \mathrm{ml}$. Baseline levels of sTNFR1 in group A were significantly higher than in group B $(p<0.01)$. Further observation of the dynamics of changes in response to injury and concomitant infection and complications showed that the highest sTNFR-1 values in group A occurred during the first examination after admission to the ED. The concentration of sTNFR1 in group B, in

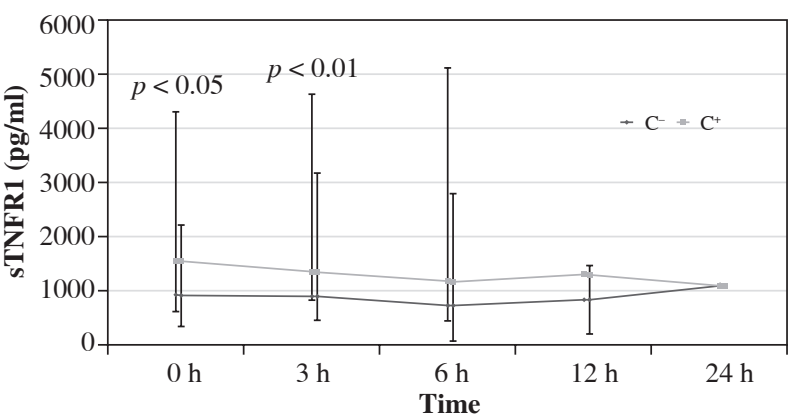

Fig. 2. sTNFR1 concentration depending on the complications 
patients with less severe injury, was not significantly different from the standard at all time points, and the highest concentration in this group was recorded in the third hour of hospitalisation (Fig. 1).

The concentration of STNFR 1 in patients with post-traumatic complications $\left(\mathrm{C}^{+}\right)$was significantly higher than in those without complications $\left(\mathrm{C}^{-}\right)$at 0 and 3 hours of observation. For the group with complications, the highest concentration value at point 0 was $\mathrm{C}^{+}$vs. $\mathrm{C}^{-}: 1561.5 \mathrm{pg} / \mathrm{ml}$ vs. $930.6 \mathrm{pg} / \mathrm{ml}, p<0.01$ (Fig. 2). The highest concentrations were observed in deceased patients (at 0 hours $-477.75 \pm 2724.01 \mathrm{pg} / \mathrm{ml}$ ). Concentration curves in both groups were similar.

Similarly to TNF- $\alpha$, statistically significant differences in concentrations were also found for STNFR 1 at 0 and 3 hours between deceased patients and survivors.

Subsequently, the correlation was investigated between cytokine levels and the values of the assessment of patient severity status according to the ISS score. The $\rho$-Spearman correlation showed a statistically significant positive relationship between baseline concentrations of STNFR1 $(r=0.59, p=0.001)$ and ISS scores and the number of complications ( $r=0.53, p=0.003)$ (Figs. 3 and 4$)$. The correlation between TNF- $\alpha$ concentration and ISS scores was significantly weaker $(r=0.38, p=0.043)$, while between the concentration of this cytokine and the number of complications it was $r=0.42, p=0.02$.

ROC curve analysis was performed in order to assess the risk of severe complications and death on the basis of cytokine concentrations (Fig. 5).

High diagnostic sensitivity calculated from ROC curves was found for sTNFR1 concentrations. Sensitivity for this cytokine was 0.8 and specificity 0.96 . It was found that sTNFR1 concentration equal to $2131.4 \mathrm{pg} / \mathrm{ml}$ correctly classified severe complications and mortality in $80 \%$ $(p=0.004)$ of cases, and the area under the curve was 0.92 . Sensitivity for TNF- $\alpha$ was 0.80 and specificity 0.83 . The cut-off point for this cytokine was $3.23 \mathrm{pg} / \mathrm{ml}$, which meant that the level of this cytokine classified mortality in $80 \%$ $(p=0.043)$ of cases; the area under the curve was 0.79 .

\section{Discussion}

Early identification of patients after severe injuries, in which a complicated clinical course may occur, is still difficult, and it has a significant impact on treatment outcomes. It should be taken into account, when interpreting the results of the present research as indicators of threatening complications, that each immune response to injury and infection can be physiological or pathological. An increased and prolonged pro- and anti-inflammatory response is a pathological response that can lead to multi-organ failure [14]. However, the distinction of these reactions in the early period of immune response in patients after major trauma is still difficult, hindering early diagnostics and appropriate therapeutic approach. The analysis

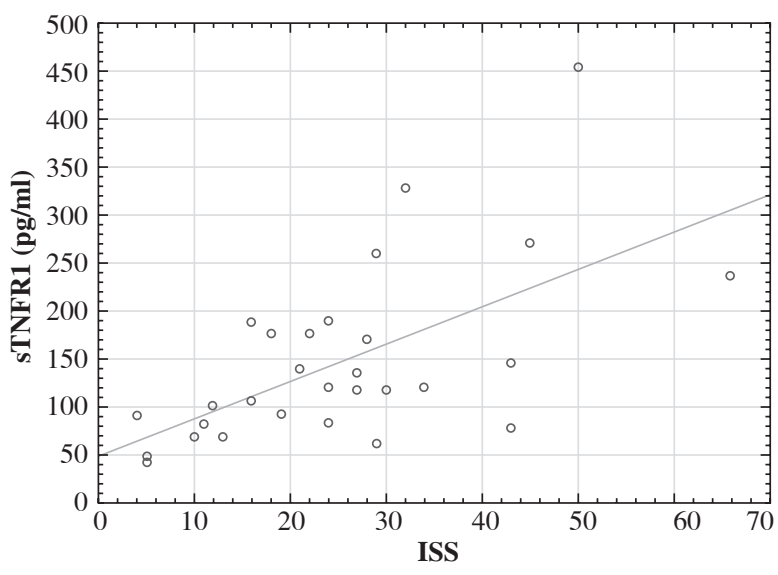

Fig. 3. Correlation plot between baseline levels of sTNFR1 and ISS scores

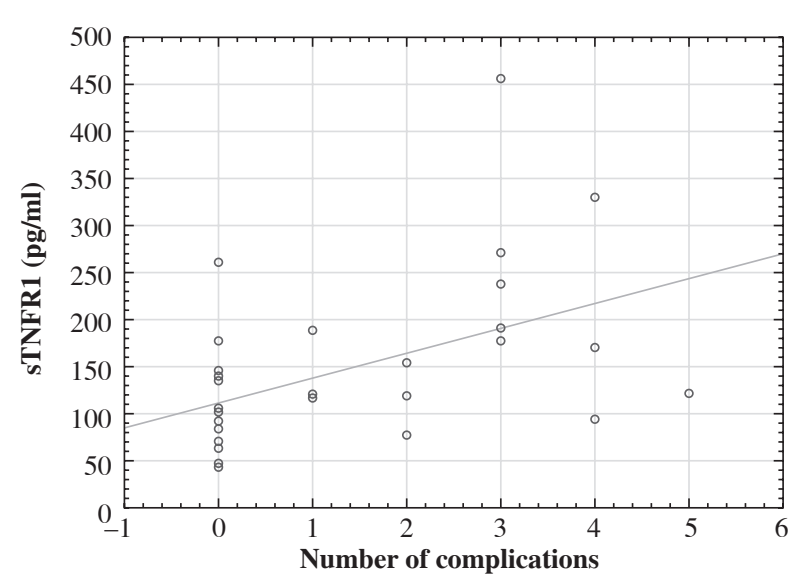

Fig. 4. Correlation plot between baseline levels of sTNFR1 and the number of complications

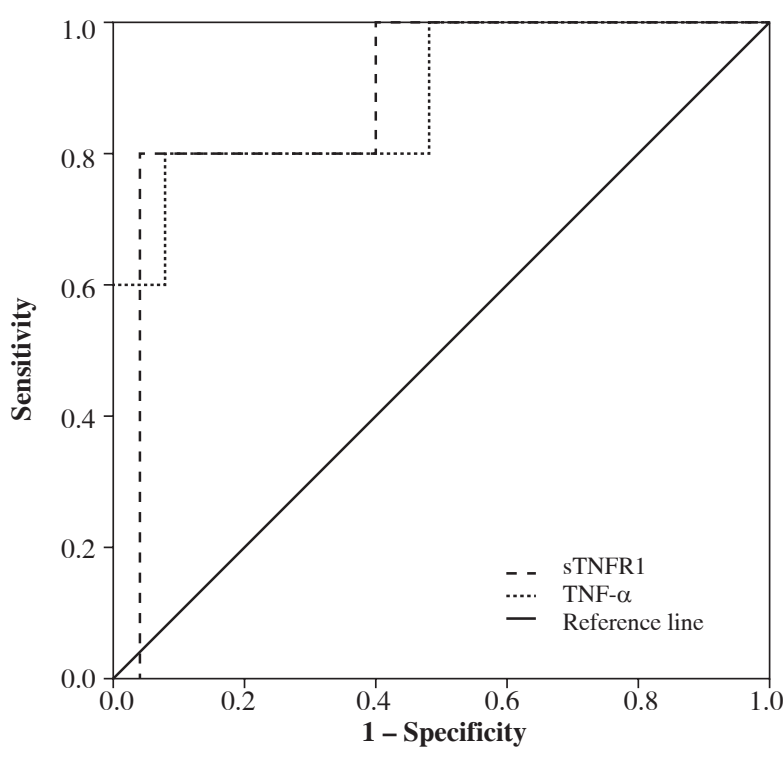

Fig. 5. Prognostic value of sTNFR1 and TNF- $\alpha$ concentrations in the group of traumatic patients (ROC curve) 
of our results in this respect indicated rapid increase in the concentration of sTNFR1 after the injury (the highest at 0 hour of the study), but only in the group after major trauma, which confirmed the occurrence of systemic inflammatory response syndrome (SIRS) in these patients. The physiological response to injury was confirmed by the results in patients without complications, in which the differences in sTNFR 1 concentrations were significant only after 0 and 3 hours of observation compared to the standard. The results of this study indicate that STNFR1 monitoring in short time intervals may be helpful in distinguishing systemic pathological response (SIRS) from the physiological response. However, it should be stressed that the "diagnostic window" was short and in the present tests "closed" as early as within three hours of admission to the ED. This was also confirmed by our previous study, in which IL-6 and IL-1Ra concentrations were tested [27].

The results of the current study indicate that the assessment of pro and antiinflammatory cytokine (TNF- $\alpha$ and sTNFR1) concentrations in the serum of post-traumatic patients may be a useful indicator facilitating the early diagnostics of SIRS, which allows us to identify the group with the highest risk of severe post-traumatic complications. Significantly higher cytokine concentrations were recorded in patients with complications, and they correlated with the extent of the injury. With the use of sTNFR1 from all patients admitted to the ED, it was possible to extract a group of patients at high risk of complications, who already had significantly elevated concentrations of this inhibitor at 0 and 3 hours after admission compared to patients without post-traumatic complications. It should be emphasised that the baseline concentrations of sTNFR1, although higher than normal, were similar in both groups $\left(\mathrm{C}^{+}, \mathrm{C}^{-}\right)$, indicating the need to monitor changes in sTNFR1 concentration, because a single test performed after the admission to the ED would have limited diagnostic value. In contrast, TNF- $\alpha$ values were significantly higher only in deceased patients, which may indicate greater usefulness of sTNFR-1, as compared to TNF- $\alpha$ in the initial period after trauma.

The analysis of cytokines performed in other centres in patients with traffic multi-organ injuries showed that the assessment of IL- 6 and IL-1Ra concentrations can be helpful in the early diagnostics of post-traumatic complications [28-32]. Similar results were obtained by Spielmann et al. [33], who assessed the risk of SIRS and/or MODS by examining cytokine serum concentrations in patients with polytrauma. Peripheral blood samples were collected from patients at the site of the accident, at the time of arrival at the hospital (30-60 minutes), and 4, 12, and 24 hours after the injury, and then once a day until the sixth day after the injury. Clinical symptoms of SIRS and MODS were monitored during the observation. Despite a variety of injuries, the occurrence of SIRS was dependent on sTNFR1 concentration, which was also confirmed by our research. In contrast to our study, there was no correlation between sTNFR1 and MODS in that study. However, as in our research, there was no significant correlation between TNF- $\alpha$ levels and the occurrence of complications, including MODS. A constant increase in the concentration of this cytokine was observed from the fourth hour of injury only in deceased patients with major trauma.

The presented research is consistent with the results from other centres [18, 33, 34]. The highest sTNFR1 levels were recorded at the time of admission to the ED in the whole group of patients. However, it should be emphasised that the level of this mediator was gradually decreasing, regardless of the patient group, but it was still at an elevated level compared to the standard. This could be due to the prevalence of a pathological inflammatory reaction to injury and infection. On the other hand, it is difficult to distinguish the physiological reaction from the pathological one in a prolonged inflammatory response, in which the concentration of the TNF- $\alpha$ inhibitor is elevated and persists for longer in the peripheral blood. Regulation mechanisms of inflammatory response are still not well understood, which has a significant impact on the diagnostic use of these parameters in critically ill patients after major trauma, but other studies have shown that cytokines are important in maintaining the increased/pathological SIRS response leading to organ damage [35].

Ribeiro et al. [34] observed that the increase in sTNFR1 concentration negatively affected the prognosis of patients after burn injury. The authors of this study found that sTNFR1 concentrations were significantly elevated in both survivors and in the deceased, but statistically significant differences in sTNFR1 concentrations between groups were recorded. In addition, a high correlation was found between sTNFR1 and the extent of burns and the APACHE II scale. However, the use of TNF- $\alpha$ as a prognostic indicator in this group of patients did not meet practical expectations. Nonetheless, the TNF- $\alpha$ concentration correlated with burn area 12 hours after the injury. The study schedule adopted by the authors $(0,6,12$, and 24 hours) was also focused on the early innate immune response, while to a lesser extent on the analysis of reactions to concomitant infections, the intensity of which usually increases in later days [35]. It is known that elevated levels of TNF- $\alpha$ occur primarily after injuries (communication injuries, burns, surgery) and in sepsis. These injuries may have a similar cytokine release profile [34]. Elevated level of TNF- $\alpha$ may be an indicator of poor prognosis [33, 34].

Other studies, in which patients were divided according to the ISS score, with similar (to ours) time intervals of blood sample collection $(0,2,6$, and $8 \mathrm{~h})$, indicated that there might be a correlation between ISS and TNF- $\alpha$ and sTNFR1 concentrations in the peripheral blood [18]. There was also a significantly elevated level of TNF- $\alpha$ as early as two hours after injury in patients with ISS $\geq 16$ compared to the control group. The highest TNF- $\alpha$ levels were 
recorded within 24 hours of the injury, and they persisted until the third day and subsequently decreased to normal concentrations. Changes in the concentration of sTNFR-1, analysed in parallel, were similar, but they persisted longer at an elevated level. Concentrations of STNFR1 were significantly higher than those of TNF- $\alpha$. Unfortunately, attempts to treat these patients with monoclonal antibodies were unsuccessful [12, 36, 37].

Analysis of the receiver operating characteristic (ROC) curve performed in the next stage of the study indicated that the observed immunological indicators (TNF- $\alpha$ and sTNFR1) could be promising prognostic markers of threatening complications and mortality. Based on the analysis of ROC curves, it was found that threshold values for the studied cytokines deviated significantly from the norm only in the case of sTNFR1. The sensitivity and specificity of the examined indicators was comparably high. It should be emphasised that commercially available immunoenzymatic tests facilitate the determination of peripheral blood cytokines in post-traumatic patients. These tests can be performed at the patient's bed, and they simplify monitoring of changes in cytokine concentrations directly after the injury, before complications occur, and during treatment. As shown by previous studies, the results of cytokine assays correlated with the level of procalcitonin [38]. The monitoring of concentrations of interleukins is more sensitive because their synthesis precedes elevated levels of CRP, procalcitonin, and other markers used in routine sepsis diagnostics and other post-traumatic complications [39, 40]. However, confirming the clinical usefulness of evaluating concentrations of these markers (TNF- $\alpha$ and sTNFR1) requires further studies in larger groups of patients treated in the ED. It is known that the cytokine response to trauma varies and thus depends on many factors (e.g. time of the test, type and extent of injury, infectious complications, and treatment), but the detection of early pathological SIRS response in routine procedures should be based on simple and easily available tests $[17,27]$. Assessing the so-called "gene storm" associated with major trauma/sepsis requires advanced diagnostic techniques that are difficult for routine use, and this explains the "cytokine storm" mechanism underlying post-traumatic complications [10, 41, 42]. The results of genetic testing for early identification of patients after major traumas with high risk of complications are promising [42].

\section{Conclusions}

Our results show that the elevated level of sTNFR1, determined in the peripheral blood shortly after injury (until 3 hours), is significantly associated with the occurrence of later complications, which in some patients lead to death. In turn, high levels of TNF- $\alpha$ shortly after injury are associated with high mortality. The value of monitor- ing concentrations of these cytokines in a selected group of patients with an increased risk of serious complications (ISS $\geq 20$ ) is promising, but it requires further research in a larger group of patients. In addition to the primary treatment, early cytokine response, within the first three hours of hospitalisation, should be considered as a potential target for therapeutic intervention in patients treated in the ED, burdened with the highest risk of death.

The authors declare no conflict of interest.

\section{References}

1. Wafaisade A, Lefering R, Bouillon B, et al. (2011): Epidemiology and risk factors of sepsis after multiple trauma: an analysis of 29,829 patients from the Trauma Registry of the German Society for Trauma Surgery. Crit Care Med 39: 621-628.

2. Angeletti S, Dicuonzo G, Fioravanti M, et al. (2015): Procalcitonin, MR-Proadrenomedullin, and Cytokines Measurement in Sepsis Diagnosis: Advantages from Test Combination. Dis Markers 2015: 951532.

3. Gogos CA, Drosou E, Bassaris HP, Skoutelis A (2000): Pro-versus anti-inflammatory cytokine profile in patients with severe sepsis: a marker for prognosis and future therapeutic options. J Infect Dis 181: 176-180.

4. Tseng CC, Fang WF, Leung SY, et al. (2014): Impact of serum biomarkers and clinical factors on intensive care unit mortality and 6-month outcome in relatively healthy patients with severe pneumonia and acute respiratory distress syndrome. Dis Markers 2014: 804654

5. Bone RC (1996): Sir Isaac Newton, sepsis, SIRS, and CARS. Crit Care Med 24: 1125-1128.

6. Tschoeke SK, Ertel W (2007): Immunoparalysis after multiple trauma. Injury 38: 1346-1357.

7. Namas R, Ghuma A, Hermus L, et al. (2009): The Acute Inflammatory Response in Trauma/Hemorrhage and Traumatic Brain Injury: Current State and Emerging Prospects. Libyan J Med 4: 97-103.

8. van Griensven M, Krettek C, Pape HC (2003): Immune reaction after Trauma. Eu J Trauma 4: 181-192.

9. Murphy TJ, Paterson HM, Mannick JA, Lederer JA (2004): Injury, sepsis, and the regulation of Toll-like receptor responses. J Leukoc Biol 75: 400-407.

10. Xiao W, Mindrinos MN, Seok J, et al. (2011): A genomic storm in critically injured humans. J Exp Med 208: 2581-2590.

11. Cuenca AG, Gentile LF, Lopez MC, et al. (2013): Development of a genomic metric that can be rapidly used to predict clinical outcome in severely injured trauma patients. Crit Care Med 41: 1175-1185.

12. Liu Q, Zhou YH, Yang ZQ (2016): The cytokine storm of severe influenza and development of immunomodulatory therapy. Cell Mol Immunol 13: 3-10.

13. Hotchkiss RS, Monneret G, Payen D (2013): Sepsis-induced immunosuppression: from cellular dysfunctions to immunotherapy. Nat Rev Immunol 13: 862-874.

14. Binkowska A, Michalak G, Słotwiński R (2015): Current views on the mechanisms of immune responses to trauma and infection. Centr Eur J Immunol 40: 206-216. 
15. Brochner AC, Toft P (2009): Pathophysiology of the systemic inflammatory response after major accidental trauma. Scand J Trauma Resusc Emerg Med 17: 43.

16. Gentile LF, Cuenca AG, Philip A, et al. (2012): Persistent inflammation and immunosuppression: A common syndrome and new horizon for surgical intensive care. J Trauma Acute Care Surg 72: 1491-1501.

17. Papaneophytou CP, Kontopidis GA (2012): Optimization of TNF- $\alpha$ overexpression in Escherichia coli using response surface methodology: Purification of the protein and oligomerization studies. Protein Expr Purif 86: 35-44.

18. Liu C, Tang J (2014): Expression levels of tumor necrosis factor- $\alpha$ and the corresponding receptors are correlated with trauma severity. Oncol Lett 8: 2747-2751.

19. Gerlach H, Gerlach M, Clauss M (1993): Relevance of tumour necrosis factoralpha and interleukin1alpha in the pathogenesis of hypoxiarelated organ failure. Eur J Anaesthesiol 10: 273-285.

20. Vandenabeele P, Declercq W, Beyaert R, Fiers W (1995): Two tumour necrosis factor receptors: structure and function. Trends Cell Biol 5: 392-399.

21. Goodwin RG, Anderson D, Jerzy R, et al. (1991): Molecular cloning and expression of the type 1 and type 2 murine receptors for tumor necrosis factor. Mol Cell Biol 11: 3020-3026.

22. Roquilly A, Villadangos JA (2015): The role of dendritic cell alterations in susceptibility to hospital-acquired infections during critical-illness related immunosuppression. Mol Immunol 68: 120-123.

23. Shelhamer MC, Rowan MP, Cancio LC, et al. (2015): Elevations in inflammatory cytokines are associated with poor outcomes in mechanically ventilated burn patients. J Trauma Acute Care Surg 79: 431-436.

24. Champion HR, Copes WS, Sacco WJ, et al. (1990): The Major Trauma Outcome Study: establishing national norms for trauma care. J Trauma 30: 1356-1365.

25. Rowell SE, Barbosa RR, Diggs BS, Schreiber MA (2011): Specific abbreviated injury scale values are responsible for the underestimation of mortality in penetrating trauma patients by the injury severity score. J Trauma 71: S384-S388.

26. Leung GK, Chang A, Cheung FC, et al. (2011): The first 5 years since trauma center designation in the Hong Kong Special Administrative Region, People's Republic of China. J Trauma 70: 1128-1133.

27. Binkowska A, Michalak G, Pilip S, et al. (2018): The diagnostic value of determination of selected cytokines in patients after major trauma - a preliminary report. Centr Eur J Immunol 43: 33-41.

28. Raymondos K, Martin MU, Schmudlach T, et al. (2012): Early alveolar and systemic mediators relase in patients at different risks for ARDS after mulitple trauma. Injury 43: 189-195.

29. Hur J, Yang HT, Chun W, et al. (2015): Inflammatory Cytokines and Their Prognostic Ability in Cases of Major Burn Injury. Ann Lab Med 335: 105-110.

30. Kirchhof C, Biberthaler P, Mutscler WE, et al. (2009): Early down - regulation of the pro-inflammatory potential of monocytes is correlated to organ dysfunction in patients after severe multiple injury: a cohort study. Crit Care 13: 1-11.

31. Strecker W, Gebhard F, Rager J, et al. (2002): Interleukin-6 (IL-6) - an Early Marker of Chest Trauma. Eur J Trauma 28: $75-84$.

32. Reikeras O, Borgen P, Reseland JE, et al. (2014): Changes in serum cytokines in response to musculoskeletal surgical trauma. BMC Res Notes 7: 128.
33. SpielmannS, Kerner T, Ahlers O, et al. (2001): Early detection of increased tumor necrosis factor alpha (TNF $\alpha$ ) and soluble TNF receptor protein plasma levels after trauma reveals associations with the clinical course. Acta Anaesthesiol Scand 45: 364-370.

34. Ribeiro CA, Andrade C, Polanczyk CA, et al. (2002): Association between early detection of soluble TNF-receptors and mortality in burns patients. Intensive Care Med 28:472-478.

35. Lier H, Böttiger BW, Hinkelbein J, et al. (2011): Coagulation management in multiple trauma: a systematic review. Intensive Care Med 37: 572-582.

36. Doessegger L, Banholzer ML (2015): Clinical development methodology for infusion-related reactions with monoclonal antibodies. Clin Transl Immunology 4: e39.

37. Neanuber C, Zeckey C, Andruszkow H, et al. (2011): Immunomodulation in Polytrauma and Polymicrobial Sepsis - Where Do We Stand. Recent Pat Inflamm Allergy Drug Discov 5: 17-25.

38. Chaemsaithong P, Romero R, Korzeniewski SJ, et al. (2016): A rapid interleukin- 6 bedside test for the identification of intra-amniotic inflammation in pretern labor with intact membranes. J Matern Fetal Neonatal Med 29: 349-359.

39. Liu Y, Hou J, Li Q, et al. (2016): Biomarkers for diagnosis of sepsis in patients with systemic inflammatory response syndrome: a systematic review and meta-analysis. SpringerPlus 5: 2091.

40. Schlosser HG, Volk HD, Splettstösser G, et al. (2007): A New Qualitive Inerleukin+6 Bedside Test Can Predict Pneumonia in Patients With Severe Head Injury-Comparison to the Standard Immulite Test and Semiquantitative Bedside Test. J Neurosurg Anesthesiol 19: 5-9.

41. Gentile LF, Cuenca AG, Vanzant EL, et al. (2013): Is There Value in Plasma Cytokine Measurements in Patients with Severe Trauma and Sepsis? Methods (San Diego, Calif) 61: 3-9.

42. Cuenca AG, Gentile LF, Lopez MC, et al. (2013): Development of a genomic metric that can be rapidly used to predict clinical outcome in severely injured trauma patients. Crit Care Med 41: 1175-1185. 